\title{
Erratum to: Enhanced insulin sensitivity and acute regulation of metabolic genes and signaling pathways after a single electrical or manual acupuncture session in female insulin-resistant rats
}

\author{
Anna Benrick • Manuel Maliqueo $\cdot$ Julia Johansson · \\ Miao Sun • Xiaoke Wu $\cdot$ Louise Mannerås-Holm • \\ Elisabet Stener-Victorin
}

Published online: 14 November 2014

(C) Springer-Verlag Italia 2014

Erratum to: Acta Diabetol

DOI 10.1007/s00592-014-0645-4

Unfortunately, the original publication of this paper contained error in the presentation of Fig. 3. In Fig. 3d, the type of stimulation should be manual stimulation. The correct Fig. 3 is given below.

The online version of the original article can be found under doi:10.1007/s00592-014-0645-4.

A. Benrick · M. Maliqueo · J. Johansson · M. Sun .

E. Stener-Victorin $(\square)$

Department of Physiology, Institute of Neuroscience and

Physiology, Sahlgrenska Academy, University of Gothenburg,

Box 434, 40530 Göteborg, Sweden

e-mail: elisabet.stener-victorin@neuro.gu.se

M. Sun $\cdot$ X. Wu $\cdot$ E. Stener-Victorin

Department of Obstetrics and Gynecology, First Affiliated

Hospital, Heilongjiang University of Chinese Medicine,

Harbin 150040, China

L. Mannerås-Holm

Department of Molecular and Clinical Medicine, Institute of Medicine, Sahlgrenska Academy, University of Gothenburg,

Göteborg, Sweden 

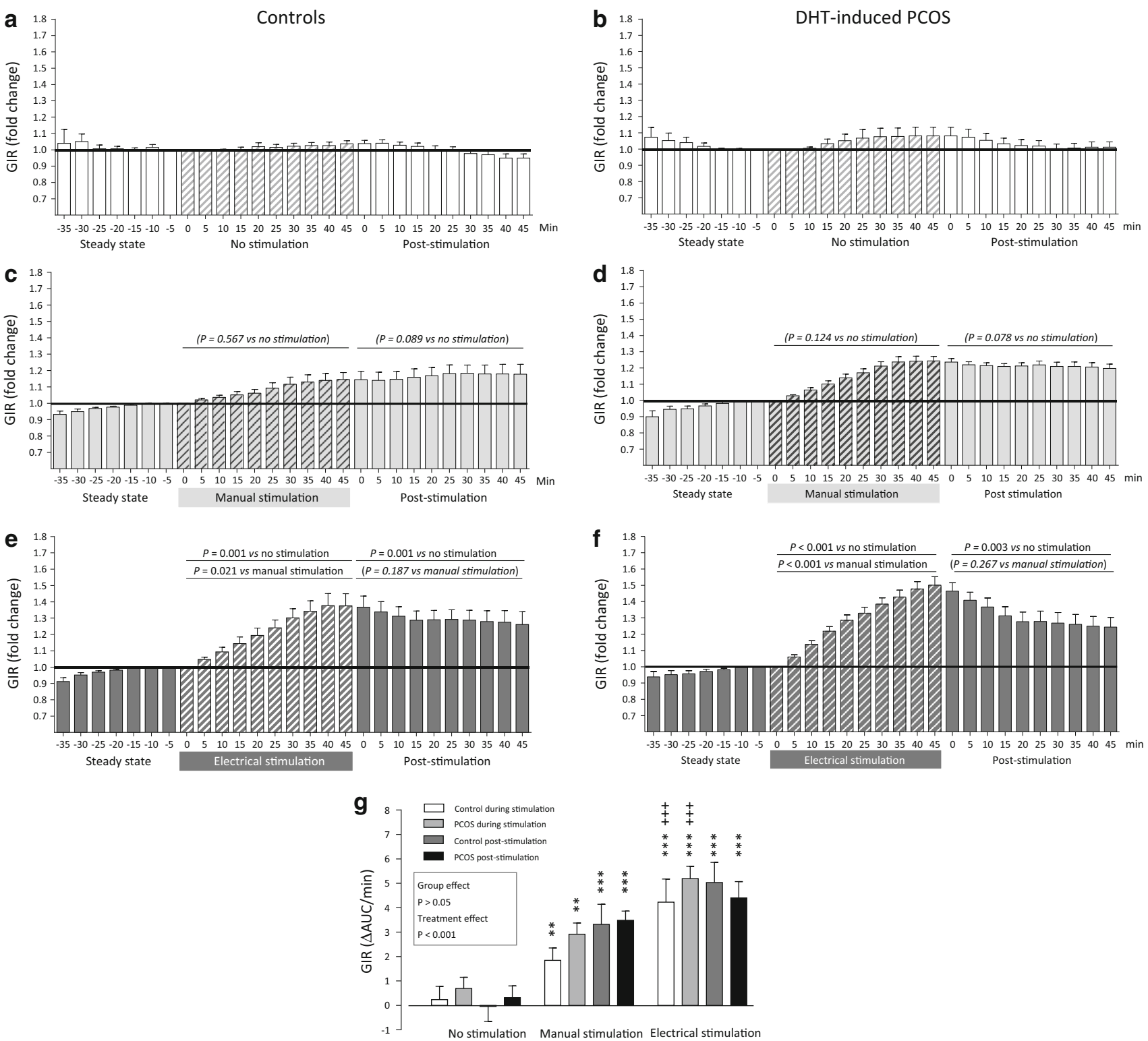

Fig. 3 Fold change in GIR at steady state, during stimulation and after stimulation in controls and PCOS rats with $\mathbf{a}$ and $\mathbf{b}$ no stimulation, $\mathbf{c}$ and $\mathbf{d}$ manual stimulation, $\mathbf{e}$ and $\mathbf{f}$ electrical stimulation. g Differences in the area under the curve $(\triangle \mathrm{AUC} / \mathrm{min})$ in controls and PCOS rats. Values are expressed as the mean \pm SEM. The differences in fold changes in GIR were determined with repeated measurement ANOVA with Bonferroni post hoc test. Changes in the AUC/min were analyzed by two-way ANOVA with the Tukey post hoc test. $* * P<0.01 ; \quad * * * P<0.001$ versus no stimulation; $+++P<0.001$ versus manual stimulation 